\title{
El Sector No Lucrativo español ante las nuevas herramientas de fundraising: el crowdfunding social, ¿antesala de la sociedad mecenas?
}

\author{
Antonia Sajardo Moreno ${ }^{1}$ \\ Departamento de Economía Aplicada \\ Instituto Universitario Polibienestar \\ Universitat de València \\ Salvador Pérez Sempere \\ Doctorando en Economía Social \\ Universitat de València
}

DOI: $10.1387 /$ reves. 17878

Fecha de entrada: 04/04/2016

Fecha de aceptación: 03/06/2016

Sumario: 1. Introducción. 2. Revisión de la literatura. 3. Metodología del trabajo empírico. 4. Análisis Cualitativo: principales resultados. 5. Análisis Cuantitativo: el crowdfunding social en España. 5.1. Plataforma Microdonaciones.hazloposible.org. 5.2. Plataforma migranodearena.org. 6. Conclusiones y retos. 7. Referencias Bibliográficas.

\section{Resumen:}

El Sector No Lucrativo en España se ha venido desarrollando tradicionalmente al abrigo del apoyo público, tanto para el desarrollo de sus actividades, como respecto de su propio mantenimiento interno. Durante la profunda crisis económica, vivida desde 2007, el retroceso que éste ha sufrido en el volumen de aportaciones, procedentes tanto de las arcas públicas, como de las donaciones privadas, así como la mayor reticencia financiadora por parte de la banca tradicional, lo han situado, de facto, en un escenario de profunda debilidad financiera. Consecuentemente, su rol como fuerza económica y social se ha visto sustancialmente reducido. Todos estos factores, le han avocado a la búsqueda de un nuevo marco de financiación, un marco diferente que surge en el mismo seno del nuevo paradigma digital en el que la ciudadanía del siglo XXI se halla inmersa, y del cual emergen nuevos y originales espacios para el desarrollo de la filantropía. El mayor exponente en estos espacios lo constituye el crowdfund-

1 Mail de correspondencia: antonia.sajarado@uv.es 
ing, que propicia el surgimiento de colectivos de ciudadanos financiadores en torno a en torno a valores y objetivos concretos y afines. En este contexto se asiste al nacimiento de un nuevo concepto que define certeramente este novedoso modelo filantrópico: la sociedad mecenas. El objeto de este trabajo es analizar las potencialidades del crowdfunding que justifican su opción por el Sector No Lucrativo, como alternativa de fundraising en la financiación de su proyectos sociales.

\title{
Palabras clave:
}

Sector No Lucrativo, filantropía, crowdfunding social, fundraising, crowdsourcing.

\begin{abstract}
:
The Non-Profit Sector in Spain has been traditionally developing with support from the public, both for the development of their activities, as for its own internal maintenance. During the profound economic crisis experienced since 2007, the decline that it has suffered in the volume of contributions, both from public coffers, and also private donations, as well as the great reluctance to provide funding by the traditional banking system, have placed it, de facto, in a scene of profound financial weakness. As a result, its role as an economic and social force has been substantially reduced. As a result of these factors, they have been obliged to search for a new funding framework, a different framework that arises in the same heart of the new digital paradigm in which citizens of the XXI century is immersed, and from which have emerged new and original spaces for the development of philanthropy. The greatest exponent of these spaces, crowdfunding, which is conducive to the emergence of groups of citizen funders in around values and goals and the like. In this context we are witnessing the birth of a new concept that defines and accurately describes this new model of philanthropy: the society patrons. The object of this work is to analyze the potential of crowdfunding as the justifiable choice for the NonProfit Sector, as an alternative fundraising technique in the financing of its social projects.
\end{abstract}

\section{Key words:}

Nonprofit Sector, Philanthropy, Social Crowdfunding, Fundraising, Crowdsourcing.

\section{Résumé:}

Le secteur à but non-lucratif en Espagne s'est développé traditionnellement grâce au soutien public, autant pour le développement de ses activités que pour son propre fonctionnement interne. Au cours de la profonde crise économique vécue par le monde depuis 2007, le déclin que ce secteur a souffert dans ses contributions financières/trésorerie/ de ses fonds, à la fois provenant des caisses publiques, des dons privés, ainsi qu'une réticence 
majeure du financement des banques traditionnelles, a situé au secteur dans une situation de faiblesse financière profonde. Par conséquent, son rôle en tant que force économique et sociale a été considérablement réduit. Tous ces facteurs ont mené le secteur à la recherche d'un nouveau cadre de financement, un cadre différent qui émerge au cœur du nouveau paradigme des technologies de l'information, où on peut trouver des nouvelles et originales formes de conduire des activités philanthropiques. L'exemple le plus notable de ces nouveaux moyens est le crowdfunding, ce qui favorise l'émergence de groupes sociaux, à valeurs et objectifs similaires, qui sont prêts à financer ces nouvelles initiatives. Dans ce contexte, nous assistons à la naissance d'une nouvelle vision qui définit un nouveau modèle de financement : la société comme son propre mécène. L'objectif de cet article est d'analyser le potentiel du crowdfunding et de justifier son rôle dans le secteur à but non-lucratif, c'est à dire dans le financement de projets sociaux de façon différente aux modalités traditionnelles.

\section{Mots-Clés:}

Secteur sans but lucratif, philanthropie, crowdfunding sociale, fundarising, crowdsourcing.

\section{Claves ECONLIT:}

D640, G23, 031, 0350.

\section{Introducción}

Las fuentes de financiación del Sector No Lucrativo en España (SNL, en adelante) han procedido tradicionalmente del Sector Público, con una dependencia superior al $50 \%$ del total de sus recursos económicos (Sajardo et al, 2007), de las aportaciones voluntarias de personas y entidades, y del apoyo financiero de la banca tradicional. Esta realidad es la base de su débil estructura financiera y de un armazón económico inseguro en el medio plazo. Esto ha condicionado históricamente tanto el potencial de actuación del SNL como su significación social y económica.

Las denominadas entidades singulares, como son Cáritas, ONCE y Cruz Roja, al igual que otras entidades de mayor tamaño, presentan un panorama diferente, al abrigo de modelos de financiación privilegiados por el Sector Público, o diversificados en base al desarrollo de actividades empresariales propias, o por el recurso a las acciones de mecenazgo y filantropía. 
La aparición y larga duración de la crisis económica que se vive en los primeros lustros del siglo XXI, ha puesto de manifiesto la debilidad de este sistema financiero y ha situado un gran número de Entidades No Lucrativas (ENLs, en adelante) ante un escenario de desamparo. Todos estos factores, le han avocado a la búsqueda de un marco diferente, al amparo del nuevo paradigma digital en el que la ciudadanía del siglo xxI se encuentra inmersa, en el que se manifiestan nuevos espacios de desarrollo de la filantropía donde las bases sociales, juegan un papel protagonista en la financiación del SNL..

En el año 2008 el 64\% de la financiación del SNL procedía de las instituciones públicas y un 36\% del sector privado ${ }^{2}$. En el año 2011 esta composición varió sus proporciones pasando a ser un $70 \%$ procedente de fondos públicos y un $30 \%$ de fondos privados. En plena crisis económica, el SNL español ha estado adecuadamente financiado hasta el año 2012, momento en el que se produce un punto de inflexión y aparecen problemas financieros reales merced a la reducción presupuestaria en todos los niveles de la Administración, y la dificultad creciente de cobro de los fondos asignados $(\mathrm{PwC}, 2014)^{3}$.

Un panorama similar viene observándose en el contexto de las ayudas del sector privado, con una reducción en la aportación de la filantropía privada tradicional ${ }^{4}$, puesto que en 2011 un 17,2\% de los españoles han finalizado, reducido o sustituido sus aportaciones a ENLs (Adeco, 2011). Asimismo se genera una disminución de los fondos procedentes de la Obra Social de las Cajas de Ahorro, pasando de un $28 \%$ en 2008 a un $21 \%$ en el año $2011^{5}$.

Adicionalmente, a nivel legislativo dos hechos cobran vital importancia, dada la coyuntura financiera de las ENLs ${ }^{6}$. Por una parte un retraso, al que no se le contempla un fin, en la adecuación de la Ley del Mecenazgo

2 Obra Social de Cajas de Ahorro, aportaciones corporativas, socios y recursos propios.

3 Una reducción del gasto público en servicios sociales, en términos globales y relativos, limitadora del potencial de acción del SNL espańol, que ha vendió conjugada con una creciente demanda, de servicios que el mismo presta. Una muestra de ello ha sido el aumento de la pobreza y la exclusión social en Espańa, desde el 23,1\% en 2008 al 26,8\% en 2012, a tenor del indicador AROPE (PTS 2012).

${ }^{4}$ La media estimada de Población donante en Espańa es del 10,3\%, frente al 33\% de media en Europa (AEFR, 2013).

5 La transformación de estas entidades financieras en Bancos, y la consecuente reducción del volumen de la obra social, ha influido negativamente en sus aportes al SNL (PwC et al. 2014).

${ }^{6}$ Según muestra el informe de la Plataforma de ONGs de Acción Social en 2012, la demora en la resolución del primero, y la activación real del segundo supondría la desaparición de más del 90\% de las entidades que componen el Tercer Sector de acción social español. 
a realidad actual, que viene esperándose, por las principales estructuras del Sector, como decisivo «balón de oxígeno» financiero, si se apuesta por un claro protagonismo de la financiación privada al Sector (PTS, 2012). Por su parte, la aparición en marzo de 2014 de un borrador de modificación de la Ley de subvenciones, en el que no parece tenerse en cuenta el contexto, la naturaleza y singularidad financiera del SNL español, al establecerse, entre otros aspectos, un límite subvencionador el 75\% del coste (art.19), o la excepción de los pagos a cuenta (art. 34), obligándole de facto a la cofinanciación del $25 \%$, o el recurso directo por el crédito.

Un último elemento significativo a este nivel ha sido la reciente sentencia de 9 de julio del 2015 del Tribunal Supremo, que declara nulo el Real Decreto 535/2013 por el que se convocaban las subvenciones de régimen general para el apoyo a los gastos de estructura de las ENLs en el citado año. Este hecho ha paralizando la recepción de alrededor de 10,5 millones de $€$, y afectando directamente al $90 \%$ de las entidades del Sector.

Toda esta situación se produce en un marco de transformación tecnológica que ha calado profundamente en el modo de comportamiento de la ciudadanía y en su forma de relación con el resto y con su propio entorno. Se trata de una realidad marcada por el uso de las herramientas digitales que está transformando profundamente el mundo en que vivimos, desplegando un amplio abanico de herramientas y recursos, uno de cuyos ámbitos de expresión es el financiero. Así, se abren ante el SNL nuevas oportunidades desde el punto de vista organizativo y operativo, para implementar mejoras en sus flujos de financiación, de información y en sus organigramas funcionales, cuya existencia no pueden permitirse el lujo de obviar (Morales, 2009); (Morales \& Ariza-Montes \& Múñiz, 2012).

En este contexto emergen herramientas de financiación novedosas para el SNL, como es el crowdfunding, que se revelan retadoras para su funcionamiento, el desarrollo de sus actividades, y en límite para su continuidad. El crowdfunding, si bien es un modelo de captación de recursos utilizado por la humanidad en numerosas ocasiones (Davies, 2014) aparición de las herramientas sociales ${ }^{8}$ y la automatización de los pagos online $e^{9}$ cuando se ha producido el despliegue real del mismo para la obtención de recursos.

7 Uno de los primeros hitos históricos del crowdfunding hunde sus raíces en la Norteamérica del siglo xviI, financiando la publicación de «The Penniless Pilgrimage» de John Taylor en 1618.

${ }^{8}$ Facebook, Twitter, Google+ entre otras.

9 Las herramientas más utilizadas en el crowdfunding son Paypal, Amazon Payments, o WePay. 
El crowdfunding es concebido como una herramienta dirigida principalmente al logro de la financiación necesaria para la puesta en funcionamiento de proyectos empresariales y sociales en un plazo temporal concreto, y generalmente breve. En el ámbito social (y de manera relevante en la ayuda al desarrollo), el crowdfunding, permite en términos cuantitativos una ampliación de su propia base social (número de individuos financiando un proyecto), pero también cualitativamente, por el acceso de múltiples colectivos vinculados o participando de alguna forma en los proyectos (Cejudo \& Ramil, 2013).

El crowdfunding es concebido como un proceso de financiación colectiva en base a aportaciones individuales de una gran masa de participantes, que configuran una red dirigida a la obtención de recursos, ya sean financieros o de otra índole, para posibilitar la realización de un proyecto concreto (Belleflame \& Lambert \& Schwienbacher, 2010, 2014) Las diferentes fórmulas operativas de este fenómeno, que van desde la mera donación de recursos, hasta la participación directa en el capital de una iniciativa concreta, pasando por préstamos entre pares, le ha dotado de un potencial de crecimiento importante a nivel mundial, experimentando un ritmo de crecimiento superior al $200 \%$ anual. De hecho, cada vez más, esta modalidad de captación de recursos es utilizada en diferentes campos de la actividad económica como podría ser la cultura, el real state, los deportes, financiación de proyectos personales y otras muchas más áreas.

En España, el fenómeno del crowdfunding hizo su aparición allá por 2010, produciéndose su despegue definitivo a partir del año 2012. Se presenta, en el SNL, como una herramienta alternativa de fundrasing, que despliega un abanico de nuevas opciones de acceso a recursos, no únicamente financieros, que ubica y aglutina a bases sociales, dispersas habitualmente, en torno a principios y valores representados por proyectos concretos, y da pie a su participación directa en los mismos. Una muestra más del desarrollo de esta modalidad de actividad socioeconómica es la aparición en Julio de 2013 de la Asociación Española de Crowdfunding ${ }^{10}$, que viene ejerciendo un relevante papel de lobby en favor de una regulación del Sector, una de cuyas principales líneas de actuación ha sido es trabajar en pro de nueva ley de mecenazgo que trate con mayor justicia la participación de la ciudadanía, con sus aportaciones individuales, en la financiación y desarrollo de proyectos sociales concretos.

10 El sitio web de la asociación puede consultarse en http://web.spaincrowdfunding.org/ 
En este contexto, el objetivo último de este trabajo es analizar las potenciales del crowdfunding para la financiación de los proyectos sociales del SNL en España, como instrumento alternativo a la financiación tradicional en el seno de la denominada nueva era de la filantropía, la era de la «sociedad mecenas» (Sajardo, 2014b). Como objetivo intermedio se persigue determinar las bases que justificaría la utilización de este nuevo modelo financiero por las ENLs, y que avalaría su capacidad como pieza clave en el futuro de sus estrategias de fundraising.

Tres son los aspectos concretos que van a ser analizados en el seno de este trabajo. Primero, identificar las principales variables que intervienen en el proceso financiador, y seleccionar aquellas que tienen un peso específico más relevante como elementos explicativos de las motivaciones y comportamientos necesarios que determinan la utilización de la herramienta de financiación del crowdfunding por las ENLs. Segundo, evaluar la escala de los proyectos sociales susceptibles de tener éxito en el seno de las campañas de crowdfunding, medir su volumen, evolución y tendencias, y a la postre su viabilidad como herramienta financiera del SNL. Y tercero, analizar las percepciones que sobre el fenómeno del crowdfunding tienen los diferentes agentes implicados en el mismo.

La metodología utilizada para la elaboración del presente artículo se fundamenta en la realización de un trabajo empírico de naturaleza mixta. De un lado, la perspectiva cuantitativa se ha basado en el análisis de las bases de datos procedentes de las principales plataformas de crowdfunding que las ENLs españolas emplean para la financiación de sus proyectos sociales, bien usando agregadores de proyectos, bien información ad hoc minuto a minuto de las plataformas, a las que han tenido acceso los autores del presente estudio. De otro lado el abordaje cualitativo se ha basado en la información secundaria obtenida merced a los datos de una encuesta realizada por una de las pioneras plataformas de crowdfunding español. De la puesta en común de ambos métodos se podrán inferir los retos a que se enfrenta el modelo de crowdfunding social en España.

\section{Revisión de la literatura}

La capacidad del SNL para adaptar sus estrategias operativas generales al entorno creado por la aparición de las herramientas sociales será, sin duda, una de las características que marcará su capacidad de pervivencia y expansión. Los recursos ofrecidos por las nuevas herramientas sociales, están siendo adoptados por las ENLs, para su gestión en los diferentes ámbitos de la comunicación, relación y búsqueda de recursos tanto humanos 
como financieros, mostrando su versatilidad y adecuación a los principios que rigen esta nueva era colaborativa en la que se halla la humanidad actual (Tapscott, 2012) ${ }^{11}$.

En este contexto Lozano (2009) estudia cómo la Economía Social, en general, y el SNL, en particular, y la web 2.0 (red social), tienen mucho en común desde el punto de vista teórico debido a la existencia de una primacía de las personas frente al capital, el interés social, la participación de los socios y la gestión democrática.

Por su lado, Murray (2010) analiza las entidades de Economía Social desde la perspectiva del nuevo paradigma tecnológico y su impacto en las tecnologías de la información, identificando aspectos básicos de alto paralelismo:

- Que se trata de una red distribuida, cuyos elementos se enlazan a través de valores compartidos, y una arquitectura organizativa federal al nivel de las industrias y del movimiento completo.

- Que se estructura en torno a valores que impulsan la importancia de la colaboración y el principio de compartir, no simplemente entre las propias entidades sino con las comunidades en las que trabajan.

- Que se organiza de manera democrática e inclusiva para todos los individuos conectados con producción tanto como clientes, como trabajadores o comunidades, a la par de que tiene una amplia experiencia exitosa en el modo de hacer efectivo este trabajo.

Huang \& Davenport (2011) desarrollaron un estudio entre los profesionales de relaciones públicas de ENLs del sector de medio ambiente, dirigido a conocer el motivo por el cual hacen, o no, uso de los social media sites (SNS). Las tareas de comunicación desarrolladas por los entrevistados, así como las percepciones sobre las SNS que tenían, fueron catalogadas en base a cuatro niveles: anuncios de información; creación de debates abiertos; gestión de las relaciones sociales, y expansión de las conexiones sociales. Los resultados muestran que la adopción de las SNS entre las ENLs

${ }^{11}$ Las potencialidades del desarrollo y comprensión de los medios sociales en general, y de Internet en particular, para la Economía Social son evidenciados por la Alianza Cooperativa Internacional en 2012 en su documento "Proyecto de una Década Cooperativa». En este documento se enfatiza el rol que los Social Media juegan en la actualidad, y sobre lo que se espera que alcancen en la próxima década, en el ámbito cooperativo. Así, la ACI, establece como una de las acciones posibles, de carácter indicativo, para alcanzar el objetivo cooperativo 2020: "Crear un proyecto de trabajo con los jóvenes adultos, los jóvenes en general y el sector de los medios sociales, para explorar la motivación de las jóvenes generaciones con respecto a las actividades de colaboración; cómo han ido cambiando y siguen modificándose la comunicación y la creación de relaciones; examinar las prácticas que han surgido en los recientes movimientos» (Mills \& Davies: 12). 
medioambientales depende de la percepción que los profesionales, a título individual, tienen de ellas. Esto ocurre si se ven como una herramienta capaz de gestionar las relaciones sociales y expandir las redes personales. Los SNS, se presentan en forma de blogs o comunidades online que animan a las personas a unirse a grupos sociales, participar en conversaciones abiertas y compartir información con grupos de usuarios de estas tecnologías grandes y heterogéneos (Donath, 2008).

Nah \& Saxton (2012) realizaron para "New Media \& Society» ${ }^{12}$ un estudio, que examinó cuáles son los aspectos que impulsan la adopción organizacional y la utilización de los medios sociales, a través de un modelo construido en base a cuatro factores clave: Estrategia, Capacidad, Gobernanza y Entorno. Así, el estudio analizó cómo las estrategias organizacionales, las capacidades, las características del sistema de gobernanza y las presiones externas juegan un papel importante en la motivación para la adopción de los medios sociales como herramienta de comunicación y relación y en los resultados de utilización de los mismos.

Utilizando Twitter, Facebook y datos de otras 100 grandes ENLs, se examinaron los factores determinantes de tres aspectos clave en la utilización de los medios sociales: adopción, frecuencia de utilización y atracción (engagement) al diálogo. El estudio contempló tres diferentes enfoques estratégicos: lobbying, fundraising e ingresos por servicios. Como conclusiones principales pueden relacionarse las siguientes:

1. Los gastos originados por el desarrollo de una estrategia basada en Lobbying tienen efectos desiguales, pero generalmente se asocia a la entidades que utilizan esta estrategia (por ejemplo Greenpeace), para su acción en los medios sociales, las actualizaciones de contenido menos frecuentes y para el uso habitual de mensajes impulsores del diálogo.

2. Aquellas entidades basadas en una estrategia de ingresos por servicios, están asociadas positivamente con la adopción y utilización de los medios sociales así como con un alto volumen de actualizaciones de su contenido. Estos resultados muestran como las entidades que optan por un sistema de generación de ingresos dirigido a pago por servicios frente a las donaciones y subvenciones, confían más en los medios sociales para facilitar la comunicación con sus clientes.

12 New Media \& Society es una revista internacional de primer nivel, que funciona en base a la revisión por pares (peer-reviewed) que publica investigaciones clave sobre comunicación, medios de comunicación, estudios culturales, sociología, geografía, antropología, economía, ciencias políticas y de la información y humanidades. http://nms.sagepub.com/ 
3. Al contrario de los que era de suponer, las entidades no lucrativas que optan por una estrategia de fundraising están negativamente relacionadas con la frecuencia de uso de los medios sociales tanto en el volumen de mensajes emitidos como el establecimiento de diálogo. Es posible que una entidad que se acostumbre a comunicar la utilización de los medios sociales pueda desplazar las actividades de fundraising realizadas offline y que resultan mucho más costosas. En este sentido las actividades tradicionales de fundraising pagadas y el envío de mensajes en los medios sociales, podrían ser sustitutivos.

La utilización por parte de las ENLs de los medios sociales parece depender igualmente de los recursos y capacidades pre existentes en ellas, especialmente de aquellos relacionados con las capacidades web (Hackler y Saxton, 2007). El factor que mayor relevancia presenta a la hora de predecir la utilización de los medios sociales es la existencia de una web previa. Queda en segundo plano, con una ponderación mucho menor en el efecto predictor, la cantidad de tiempo transcurrida desde que se implementó esa web. Las capacidades que se desarrollan para la creación y mantenimiento de una web que resulte realmente influyente, conllevan efectos positivos en la adquisición de habilidades para la utilización eficiente de las nuevas herramientas tecnológicas y de la comunicación como son, por ejemplo, Facebook y Twitter.

El análisis de las características del sistema de gobernanza de las ENLs, desde el enfoque de su influencia en la predicción de la utilización de los medios sociales por parte de estas, muestra una importante influencia de esta variable. Nah \& Saxton (2012) constatan en su trabajo que «las características de gobernanza de la organización son cruciales para asegurar que los recursos son utilizados adecuadamente y las estrategias con respecto a la adopción y utilización de las tecnologías de la información» (Hackler \& Saxton, 2007:481). En este estudio se detectaron como factores determinantes para la adopción y uso de los social media la estructura como asociación (Smith, 1993), el tamaño del grupo directivo (Olson 2000), y la eficiencia organizativa (Gill \& Flynn \& Reissing, 2005).

Por otra parte, en relación a la influencia del entorno en la adopción y utilización de los medios sociales, se observó como la dependencia de donaciones de personas individuales tiene gran influencia en el modo en que las organizaciones utilizan los recursos que ofrecen los medios sociales. En contraste, la dependencia de la financiación gubernamental, no ha mostrado ser una variable que influya de un modo sustancial en este aspecto. No obstante, los resultados obtenidos en el estudio se muestran demasiado débiles para ser tomados en consideración a la hora de evaluar el grado de influencia que ejerce la financiación gubernamental en el modo en que las organizaciones utilizan estos medios. 
Por su lado Bortree \& Seltzer (2009) llevaron a cabo una investigación para detectar los resultados obtenidos merced a la utilización de la comunicación con estrategias dialógicas, a través de un muestreo intencional a 50 perfiles de Facebook, pertenecientes a entidades no lucrativas cuyo cometido principal era la defensa del medio ambiente. En este estudio se examinó de qué manera las estrategias dialógicas, utilizadas por estos grupos en la actividad de sus perfiles en las redes sociales, provocan un diálogo con un mayor grado de compromiso entre las propias organizaciones y los visitantes de sus perfiles sociales.

Como principal conclusión del estudio cabe indicar la existencia real de una relación entre los resultados dialógicos y tres estrategias dialógicas concretas, como son la conservación de los miembros pertenecientes a una comunidad, la generación de visitas recurrentes a los perfiles en Facebook, y el grado de engagement de la organización. Así, la generación de visitas recurrentes presenta una correlación, altamente significativa, con el número de respuestas que se producen entre usuarios, es decir, con la multilateralidad de la conversación. Además, se evidenció que la conservación de los visitantes a los perfiles muestra una relación recíproca con otras dos variables, a saber, el índice de crecimiento de la red y el grado de respuesta con la que la organización provee a los usuarios. Finalmente, se observó cómo el grado de engagement con la propia entidad, mantiene una relación prevalente (frente a las otras dos estrategias indicadas), con los resultados obtenidos. En otros términos, se halla positivamente relacionado con los seis resultados dialógicos que fueron detectados, que son las entradas escritas por los usuarios, la actividad que mantienen en la red, la extensión de la red social, el crecimiento de la red y la respuesta de la organización a los usuarios ${ }^{13}$.

De relevancia resulta el estudio de NTEN \& Common Knowledge \& Blackbaud (2012) ${ }^{14}$ entidades que desarrollan su actividad en torno a las

${ }^{13}$ La utilización de estrategia dialógicas para la creación de oportunidades para la involucración en el diálogo puede producir resultados positivos como es por ejemplo un incremento en el número de personas involucradas que interactúan con la organización, gracias al crecimiento de la red social de la organización.

${ }^{14}$ El estudio se realizó entre los meses de enero y febrero de 2012 en base a una encuesta online dirigida a profesionales de entidades no lucrativas de diferente (un $46 \%$ de los encuestados desarrollan su actividad en organizaciones que cuentan con un presupuesto anual de menos de un millón de USD, un 32\% entre 1 y 5 millones, 18 organizaciones entre 6 y 50 millones, 3 de ellas entre 51 y 250 , una de ellas cuenta con un presupuesto superior a los 250 millones de USD). Las preguntas se categorizaron en dos grupos primarios: aquellas dirigidas a la utilización de redes sociales comerciales (Facebook, Twitter, Google+, LinkedIn, etc), y las relativas a la utilización de redes sociales de implementación propia (es decir, construidas utilizando recursos propios de la entidad). Se obtuvo la participación de 3522 profesionales, invitándolos vía email a una encuesta online. 
entidades sin ánimo de lucro, que desde el 2009 realizan una serie de estudios similares sobre las tendencias y comportamientos en torno a las redes sociales como parte de la actividad de las entidades no lucrativas en los apartados de marketing, comunicaciones, fundraising y utilización de las Tecnologías de la Información. Este estudio confirmó un continuo crecimiento en la importancia que otorgan los profesionales de las entidades no lucrativas a las redes sociales, y cómo los principales medios sociales (Facebook, Twitter) están consolidando su liderazgo y ampliando su dominio sobre el resto de medios sociales.

Entre sus principales conclusiones cabe extraer del citado trabajo son: 1) la táctica más común de fundraising en Facebook es la solicitud de donaciones personales. El $46 \%$ de los usuarios que contempla la utilización de Facebook con estos fines, con el siguiente nivel de incidencia: donaciones individuales $(33 \%)$, promoción de eventos $(20 \%)$, financiación de causas concretas $(17 \%)$, personal fundraising $(11 \%) ; 2$ ) el tamańo de los presupuestos asignados por las entidades a las labores en las redes sociales y el personal dedicado para ello sigue creciendo; 3) los principales factores que permiten catalogar de exitosa la labor en las redes sociales son el establecimiento de una estrategia adecuada $(41 \%)$, la priorización por el equipo directivo de la labor en las redes sociales (37\%), y la dedicación de personal para la labor en las redes sociales(28\%); 4) las redes sociales construidas internamente por cada organización siguen manteniéndose en un $13 \%$, creciendo el promedio de sus miembros en un $265 \%$ anual, siendo desarrolladas a través de software libre, tanto a nivel de Sistemas Operativos (LINUX), servidores web (Apache), o CMS (Drupal, Joomla, Wordpress); 5) las inversiones de las ENLs en redes sociales se justifican todavía de un modo muy amplio en el impacto programático (soft ROI) ${ }^{15}$. Un pequeño porcentaje de los encuestados justifican la dedicación de recursos en base al retorno de la inversión (hard ROI).

El estudio de Achieve (2013) ${ }^{16}$ dirigido al análisis e interpretación del modo en que los Millennials ${ }^{17}$ conectan y se comunican con aquellas iniciativas, desarrolladas por ENLs, que producen un impacto emocional en ellos confirma el hecho de que esta generación cuenta con una creciente

15 El Soft ROI se define como un retorno basado en activos intangibles - credibilidad, branding, engagement.

16 La metodología utilizada para la realización de esta investigación se ha basado en una encuesta, realizada online, y distribuida a los Millennials a través de 14 entidades colaboradoras en la investigación. Adicionalmente se han realizado test de usabilidad en los diferentes elementos utilizados, por parte de nueve ENL, como elementos de presencia online: página web, páginas en facebook y twitter, correos electrónicos y herramientas de movilidad.

17 Personas nacidas entre 1980 y 2000. 
inquietud para conectar, involucrarse como voluntarios y hacer donaciones a aquellas causas con las que se sienten identificados.

En idéntico sentido Gerber \& Hui \& Kuo (2011) observan las motivaciones que encuentran los participantes en procesos de crowdfunding a la hora de involucrarse en ellos. Igualmente Greener \& Hui \& Gerber (2013) trata los intercambios que se producen en todo procesos de crowdfunding más allá de los meramente económicos, y de qué modo el diseño de las plataformas puede influir en ello.

Un prisma diferente es el que aborda Mollick (2014), ubicada en la perspectiva del promotor de proyectos para estudiar las dinámicas de funcionamiento del crowdfunding. En este punto Mosseyev (2013) estudia el impacto que la utilización de los medios sociales tiene en el proceso de crowdfunding e hipotetiza sobre las relaciones existentes entre variables del tipo número de seguidores con los resultados de las campañas de financiación.

En todo proyecto de crowdfunding confluyen diferentes sujetos que desarrollan roles complementarios, todos ellos necesarios, para la realidad del mismo (Zivilichovsky, Inbar, Barzilay 2014). De un lado nos encontramos a la entidad que desarrollo el proyecto y que precisa de la financiación necesaria para poder llevarlo a cabo. Por otra parte el colectivo de personas que, de un modo u otro, llegan a entrar en contacto con el proyecto, sienten afinidad por él, y deciden apoyarlo económicamente. La motivación final que impulsa a los donantes a participar en la financiación de proyectos de terceros, reside en la recepción de una recompensa. Esta puede constar de un valor inmaterial de naturaleza psicológica o material en base a bienes no económicos.

Finalmente, un tercer sujeto, la plataforma prestadora de servicios de crowdfunding, que es quien posibilidad la puesta en contacto de los otros dos actores de la campaña de crowdfunding. Para ello proporciona la asesoría necesaria, las herramientas de gestión de las aportaciones, así como de promoción del proyecto a través de diferentes herramientas sociales.

El crowdfunding es un fenómeno que, ulteriormente a la obtención de recursos para el desarrollo de proyectos concretos, presenta un conjunto de principios y formas de trabajo que posibilitan ese acercamiento entre la sociedad y los proyectos sociales que apoya (Hemer 2011):

1. Creación de comunidades de soporte en torno a proyectos y no en torno a entidades.

2. Aplicación de la inteligencia colectiva, siguiendo la lógica del crowdsourcing, dirigida al impulso de un proyecto social común.

3. Aparición de la figura del prosumer, bajo la cual el ciudadano participa activamente en los proyectos 
4. Nuevos modelos de proyectos para nuevas formas de participación (voluntariado) y fundraising (financiación).

5. Cohesión entre las diferentes roles participativos en proyectos sociales, fusionándose las figuras tradicionales con las más novedosas: voluntariado, implicación, donación, activismo, redes

La taxonomía habitual aplicada al crowdfunding varía en función del parámetro que definamos como variable. Si consideramos como elemento diferenciador el objetivo por el cual una persona participante como aportante en un proyecto , realiza efectivamente esta aportación, nos encontramos con el crowdfunding de donación y el crowdfunding de recompensa.

En el crowdfunding de donación, la motivación actúa como acicate definitivo en la participación del financiador, sin esperar ninguna gratificación más que ese a satisfacción personal que percibe por haber hecho lo correcto. Este modelo de crowdfunding es el más extendido en el ámbito de los proyectos sociales (Grifin 2012) (Bradford 2012) El otro brazo del crowdfunding sin fines monetarios es el crowdfunding de recompensa, en el que, a cambio de la aportación económica del participante, este obtiene un retorno tangible en base a cual haya sido esta. Este tipo de crowdfunding es utilizado principalmente para proyectos culturales. ${ }^{18}$

Uno de los parámetros con los que se identifica a toda campaña de crowdfunding es el montante económico solicitado para la realización del proyecto. Según la gestión del resultado Cumming \& Leboeuf \& Schwienbacher (2014), analizan dos tipos de plataformas de crowdfunding.

1. Un modelo que condiciona el acceso a los fondos por parte de los promotores de la campaña, al alcance de la totalidad del montante solicitado. Esta modalidad tiene sentido en para la financiación de proyectos concretos, en los que la viabilidad de su puesta en marcha exige unos requisitos económicos mínimos, imprescindibles.En consecuencia, en aquellos casos en los que la cantidad solicitada no es alcanzada, los fondos son devueltos a las personas que los habían comprometido con el el proyecto. Este modelo se denomina de «Todo o nada».

2. Alternativamente existe la posibilidad de recibir la suma donada, independientemente de si el montante ha cubierto el $100 \%$ de la suma solicitada. Este modelo conocido como "Todo cuenta» suele utilizarse en proyectos de un amplio campo de acción, en los que no hay un punto final del proyecto. En estos casos, toda aportación es útil.

18 Conviven en este escenario dos modelos adicionales de crowdfundng cuyo objetivo es la obtención de rendimientos económicos. Son el equity based crowdfunding y el crowdlending. Estos dos modelos quedan fuera del ámbito de estudio del presente artículo, motivo por el cual no vamos a profundizar en ellos. 
Lambert (2010) ofrece en su trabajo una revisión de las variables involucradas en los procesos de crowdfunding y un posterior análisis para determinar su influencia en la finalización exitosa de las campańas. Belleflamme et al. (2013) traza un estudio similar al anterior desde la perspectiva del crowdfunding individual, es decir, el realizado directamente sin la utilización de ninguna plataforma proveedoras de este tipo de servicios. Por último Davies (2014) delimita las características de un tipo de crowdfunding, el crowdfunding cívico, el cual grandes similitudes con el enfoque que se pretende aportar en este artículo sobre el crowdfunding social, si bien el fin último de ambas clases, es diferente.

\section{Metodología del trabajo empírico}

Con el objeto de justificar la utilización del crowdfunding como estrategia de fundraising del SNL, se ha optado por la combinación de metodología de naturaleza cualitativa y de índole cuantitativa.

La identificación de las principales variables que intervienen en el proceso del crowdfunding procede de los datos empíricos obtenidos de las principales plataformas utilizadas por las ENL españolas para la financiación de sus proyectos. A través del estudio del proceso financiador, desde la concepción de la propia idea del proyecto, hasta la ejecución del mismo, han sido seleccionadas aquellas que tienen un peso específico más relevante como elemento explicativo de las motivaciones y comportamientos necesarios que determinan la utilización de este modelo de financiación por las entidades del SNL.

Adicionalmente, mediante la recogida y análisis de datos cuantitativos procedentes de la información obtenida de las propias plataformas, se pretende evaluar la escala de los proyectos sociales susceptibles de obtener el éxito mediante rondas de financiación de crowdfunding, medir el volumen evolución y tendencias para dibujar el estado del arte del crowdfunding social y concluir sobre su viabilidad como herramienta de financiación de las ENLs, en el contexto socio-económico actual y su adecuación como parte de la solución a la continuidad e independencia de las mismas.

Para abordar el aspecto cualitativo han sido evaluadas las percepciones que sobre el fenómeno del crowdfunding tienen las distintas partes involucradas, a saber, la entidad promotora del proyecto objeto de la campańa de crowdfunding, el conjunto de personas que se han visto involucradas en campañas como donantes y las propias plataformas proveedoras de servicios de crowdfunding. El rol de estas últimas es el de agentes agregadores de información, proveniente de su propia actividad y necesaria para la realización de tal tipo de estudios. 
En este ámbito, se ha empleado la información secundaria obtenida a través de una encuesta realizada en abril de 2014 por la plataforma lanzanos. $\operatorname{com}^{19}$. La citada encuesta fue enviada a los mecenas de los distintos proyectos presentes en la citada plataforma. No obstante, al tratarse de una plataforma creada para la promoción de la creatividad a través de la tecnología ${ }^{20}$ se podrán inferir conclusiones que son válidas desde un punto de vista general. No obstante, resulta imprescindible para la validación de las citadas conclusiones, y acotar su espacio al del crowdfunding social, la realización de una encuesta similar en este último ámbito. Para ello se han mantenido conversaciones con los representantes de diferentes plataformas, ${ }^{21}$ con el objeto de conocer la existencia de encuestas similares o la disponibilidad para la realización de los mismos. Así, se ha podido constatar que la encuesta y posterior análisis, elaborado por lanzanos.es, es el primero y único existente de esta naturaleza en España. La predisposición a la elaboración de informes similares en otras plataformas, ha sido altamente positiva.

Desde el punto de vista cuantitativo, el obstáculo principal reside en el hecho de que las plataformas de crowdfunding tienen su base en registros digitales, lo que crea un universo de información difícilmente manejable por el ser humano. No obstante, en los últimos meses, han aparecido los primeros agregadores de proyectos de crowdfunding, como thecrowdfundingcentre.com o crowdsurfer.com. Estas herramientas, que han sido consultadas para la realización del presente estudio, aglutinan información procedente de la mayoría de plataformas de equity y rewards crowdfunding ${ }^{22}$, y presentan información válida para una comprensión global del fenómeno del crowdfunding.

Sin embargo, con su configuración actual no permiten dibujar claramente el estado del crowdfunding social en nuestro país. Por este motivo, en las conversaciones mantenidas con los representantes de las plataformas de crowdfunding, se ha solicitado acceso a los datos que éstas generan minuto a minuto, en base a su propia actividad. En este contexto, se ha detectado la inexistencia de un tratamiento exhaustivo de la misma, si bien, alguna de ellas ha sido capaz de facilitar un registro histórico de

19 Lánzanos.es ha sido primera plataforma de crowdfunding en España. Está activa desde diciembre de 2010.

$20 \mathrm{http}: / / \mathrm{www} \cdot l$ lanzanos.com/quienes/

${ }^{21}$ El listado de las plataformas de crowdfunding con los que se ha contactado se encuentra en el capítulo de bibliografía

22 Estas dos modalidades de crowdfunding se caracterizan por la expectativa de un retorno financiero por parte del donante, bien a modo de participación en beneficios (equity) bien por los rendimientos financieros del capital prestado (lending). 
proyectos que incluyese la información relevante para los objetivos de las plataformas y que, no coincide completamente, con los de los autores del presente trabajo.

En este contexto, se ha procedido a la extracción manual de los datos cualitativos deseados, accediendo a todos y cada uno de los proyectos presentes en las plataformas, para revisar y extraer la información pública que de ellos se muestra.

\section{Análisis Cualitativo: principales resultados}

El crowdfunding, tal y como lo concebimos hoy en día, no tendría sentido sin el soporte tecnológico de plataformas que conjugan la presentación del proyecto, la gestión de los cobros y la interacción con los medios sociales. Estos tres elementos existen dentro de un ecosistema tecnológico, captador de grandes cantidades de información, que registra en sus $\log ^{23}$ toda la información generada en cualquier interacción del usuario, ya sea mecenas promotor o mero espectador. Esta realidad pone a disposición del investigador grandes volúmenes de información de difícil manejo por el ser humano, pero que, mediante la utilización de la tecnología adecuada, permite interpretaciones, modelos y análisis que alumbren teorías concretas.

Para una correcta evaluación cualitativa de las dinámicas de funcionamiento del crowdfunding es de gran ayuda contar con la participación directa de los actores involucrados en él. Principalmente los mecenas y los promotores de los proyectos. Acceder a ellos es factible gracias precisamente al soporte tecnológico mencionado en los párrafos anteriores.

Así pues, en marzo de 2014 la plataforma lanzanos.com, realizó una encuesta de preguntas cerradas, utilizando un cuestionario $C A W I^{24}$ a 4.070 usuarios de su plataforma, de los cuales el 77,5\% (3.153) han participado como mecenas en algún proyecto de crowdfunding durante los últimos 3 años.Lanzanos.com es la primera plataforma de crowdfunding que surgió en España. Desde su aparición en el mes de diciembre de 2010 en ella se han financiado con éxito alrededor de 365 proyectos en las diferentes ca-

${ }^{23}$ Un log es un registro oficial de eventos durante un rango de tiempo particular. En el ámbito de la seguridad informática es utilizado para registrar datos o información sobre quién, qué, cuándo, dónde y por qué un evento ocurre para un dispositivo en particular o aplicación.

${ }^{24}$ Computer Assisted Web Interview 
tegorías que esta plataforma contempla ${ }^{25}$ al tratarse de una plataforma no exclusiva de crowdfunding social. No obstante, ante la inexistencia de estudios similares referidos a plataformas dirigidas a la promoción de proyectos sociales únicamente, analizamos este estudio de lanzanos.com y cruzamos sus conclusiones con artículos y la bibliografía académica encontrada, dejando abierto, para una investigación posterior, la realización de una encuesta de esta índole en el ámbito concreto del crodwfunding social. Es en este marco en el que las ENLs pueden utilizar este método alternativo de financiación para hacer frente a sus retos.

El estudio ha sido realizado por la empresa Two Much research studio ${ }^{26}$, y aporta conclusiones sobre la motivación de las personas que apoyan proyectos de crowdfunding, el valor que los mecenas buscan en un proyecto, los requisitos específicos que debe poseer un proyecto para captar la atención de la comunidad o las dudas que sobre este fenómeno tienen., incluso, los participantes en él.

De este estudio es importante resaltar el hecho de que el mecenas participa en la financiación de un proyecto a cambio principalmente de un retorno concreto y este no tiene porqué consistir en un bien material. Así pues, en base a las distintas opciones de respuestas ofrecidas en la encuesta, y teniendo en cuenta la naturaleza de lanzanos.com como plataforma no exclusiva de proyectos sociales, un 69\% de los entrevistados seleccionó la opción "contribuyo a crear lo que me gusta", un $61 \%$ porque se trata de una vía sin intermediarios y «estoy apoyando de forma directa a los creadores", un 54\% porque "contribuyo a crear cosas innovadoras» y un 51\% porque «siento que estoy apoyando el impulso emprendedor».

Filtrando estas respuestas utilizando el cedazo de la iniciativa social, es de destacar el valor de un eco de carácter, que concluye en la respuesta de «Siento que no somos tan dependientes de los poderes tradicionales». Este concepto comulga perfectamente con la necesidad de independencia por parte de las ENLs de la financiación de las administraciones sin, por supuesto, suponer una ruptura con ellas. El crowdfunding no crea la sensación de ir contra-corriente, permite sumarse al cambio desde la facilidad (lanzamos.com, 2014)

A la hora de trazar una clasificación del tipo de mecenas que participan en la financiación de proyectos de crowdfunding, se observa en el estudio que no existen diferencias decisivas que guarden una relación directa con aspectos como el perfil socio-demográfico, sexo, edad, nivel de estudios,

25 De este total de proyectos exitosos un $15 \%$ (50 proyectos) aparecen bajo la categoría solidarios, considerados por los autores del presente artículo dentro de la categoría de crowdfunding social sobre la que versa el mismo.

${ }^{26} \mathrm{http}: / /$ www.twomuchrs.com/ 
situación laboral o situación económica personal. Más bien, las diferencias en sensibilidad del mecenas se centran en la actitud desde donde éste otorga un sentido al crowdfunding.

Las dos actitudes predominantes son la denominada por los elaboradores del estudio como ChangeMaker — personas que entienden el crowdfunding como una "forma que tenemos los ciudadanos para cambiar el mundo»-y la Pragmática — que considera el crowdfunding como una fórmula que «está bien para que salgan adelante algunos proyectos con el apoyo de la gente, pero poco más».

Analizando el grado de participación el citado estudio propone la existencia de tres perfiles o sensibilidades al crowdfunding: los entusiastas (41,8\% de los encuestados), los moderados (46,5\%) y los fríos $(11,7 \%)$. Se constata que existe una relación directa entre sensibilidad y participación en los proyectos, de modo que cuanto mayor es la frecuencia de participación en proyectos, mayor es también la proporción de entusiastas y menor la de moderados y fríos.

En cuanto a la cuantía de las aportaciones, ésta no viene marcada por la pertenencia del mecenas a un grupo concreto u otro en la clasificación expuesta anteriormente, sino por el interés que suscite la idea, y por el formato del intercambio que se produzca tras la participación en su financiación. La encuesta deja entrever que las recompensas desinteresadas o basadas en donaciones cosechan aportaciones individuales muy inferiores a aquellas que plantean la entrega de un bien o un servicio.

En este sentido, coinciden las guías de éxito de diferentes plataformas de crowdfunding, concretamente referencian las pertenecientes a kikcstarter.com y startsomegood.com - dos plataformas de un peso específico relevante en el mundo del crowdfunding, generalista la primera y social la segunda que presentan como valor fundamental el contenido de la historia que contiene la idea que genera la campaña. Una tercera plataforma, fundly.com, con un peso específico importante en el ámbito del crowdfunding social ${ }^{27}$, pone su atención en tres factores que deben tomarse en consideración para alcanzar el resultado esperado en una campaña de crowdfunding: confianza, transparencia y vínculos de conexión ${ }^{28}$.

En cuanto a formato del intercambio, desde el punto de vista del crowdfunding social las ENL pueden obtener fondos más fácilmente por su no enfoque en los beneficios( Lambert et al, 2010) y el tipo de recompensas, de intercambio, ofrecido por estas suelen presentarse en tres formatos

\footnotetext{
27 http://www.forbes.com/sites/rahimkanani/2012/04/07/fundly-com-is-transforming-online-philanthropic-political-and-individual-giving/

28 Trust, Transparency and Ties
} 
diferentes: el de gratitud, de reconocimiento o el tangible (startsomegood. com, 2014).

El estudio de lanzanos.com se refiere igualmente a conceptos relacionados con la motivación de los mecenas hacia los proyectos concretos, objeto de las campańas de crowdfunding. En este sentido marca cuatro cualidades, presentes en los mismos, que definen el su potencial:

1. Que tenga una propuesta de alta calidad

2. Que su ejecución sea factible

3. Que exista una claridad en la aplicación del importe solicitado

4. Que el proyecto en sí, se muestre de un modo que genere atracción al usuario.

Analizando con más detalle las respuestas obtenidas en este estudio, encontramos en el tramo de factores a los que el usuario dota mayor importancia a la hora de decidir su participación o no en una campaña de crowdfunding valores en torno a principios de justicia social y de la innovación. Se valoran positivamente propuestas que creen riqueza y que incidan en la atención a los bienes y derechos sociales que se han visto más afectados por la política de recortes presupuestarios que estaba en plena efervescencia en la fecha de realización de la encuesta ${ }^{29}$.

Los factores que cuentan con muy poco poder de impulso a la participación por parte de los potenciales mecenas, son aquellas que contienen factores de riesgo y que generan dudas sobre su viabilidad.

Como conclusión se observa claramente que el crowdfunding cuenta con los elementos de atracción necesarios que lo hagan devenir en una herramienta habitual de financiación de proyectos sociales.

Adicionalmente a este tipo de análisis, los mecenas potenciales toman en cuenta igualmente aspectos sobre la personalidad percibida en los promotores del proyecto. La honestidad con una valoración positiva por parte del $80,9 \%$ de los encuestados, es la característica que ocupa el primer escalón esta valoración. Le sigue la creatividad con un 72,3\% y el entusiasmo con un $69,8 \%$. En su conjunto estas tres características son consideradas por los analistas del estudio como provocadoras de emoción en el proyecto.

Ocupan las siguientes posiciones, y ya a cierta distancia, la sensatez con un $47,1 \%$, el rigor $41,7 \%$, la inteligencia $37,9 \%$ y la experiencia en el oficio con un 33,1\%. La definición analítica atribuida a estos factores es la de actuar como «disolventes del riesgo» ${ }^{30}$.

29 Septiembre de 2013 y Marzo de 2014

30 concepto anotado por la empresa Two Much, realizadora del estudio 
A la hora de evaluar las áreas de duda o sombras en el crowdfunding por parte de los mecenas, se hallan reservas sobre el bajo nivel de calidad de algunos proyectos, sobre la fiabilidad de los promotores y en relación con ambas cuestiones, la pregunta sobre la responsabilidad de las plataformas en 10s proyectos que presentan o su mera actuación como comisionistas, y las garantías jurídicas que sustentan este sistema de financiación.

Habitualmente las plataformas comerciales de crowdfunding, cobran un porcentaje del importe recaudado en las campañas exitosas como márgenes comerciales para dar viabilidad a su labor. En el caso del crowdfunding social, al menos en las plataformas españolas, se encuentra un tratamiento especial en todo lo referente al crowdfunding social. Algunas plataformas cargan con un porcentaje más reducido de lo habitual a los proyectos que son considerados como solidarios ${ }^{31}$ y otras, centradas en la financiación de proyectos sociales exclusivamente, suelen no cargar ningún coste a los promotores de los proyectos.

En este segundo bloque se halla migranodearena.org, y microdonaciones.hazloposible.com, ambas sustentadas por fundaciones, lo que les permite ofrecer este servicio de un modo totalmente gratuito.

Como conclusión, el informe sobre el que pivota este apartado del artículo, concluye en una definición del crowdfunding como «un cambio hacia algo nuevo y valioso, facilitado". Un 74,5\% de los encuestados opinan que este fenómeno seguirá su crecimiento en los próximos años extendiéndose cada vez entre más gente. ${ }^{32} \mathrm{El}$ informe del primer cuatrimestre del año 2014, emitido por la plataforma thecrowdfundingcentre.com, da validez a esta percepción de los encuestados y confirma un crecimiento durante los cuatro primeros meses de este año del $101,47 \%$ a nivel mundial en todos los tipos de crowdfunding.

El estudio de naturaleza similar, centrado en los usuarios de plataformas como migranodearena.org, microdonaciones.hazloposible.org, centradas exclusivamente en proyectos con fines sociales constituye la herramienta adicional para determinar los principios motivacionales y de comportamiento en torno a los cuales el individuo medio se ve incitado a participar en la promoción de un proyecto de esta índole. Los principales resultados se recogen en el apartado siguiente.

\footnotetext{
31 lanzanos.com pasa del $5 \%$ para proyectos generales al $1 \%$ para proyectos solidarios.

$32 \mathrm{El}$ informe completo puede consultarse en http://www.lanzanos.com/uploaded/ Crowdfunding_mecenas_Conclusiones.pdf
} 


\section{Análisis Cuantitativo: el crowdfunding social en España}

Para la elaboración del componente cuantitativo de este trabajo fue solicitado el acceso a la información facilitada directamente por las propias plataformas ${ }^{33}$ seleccionando en ella aquellos proyectos que cuentan con una componente social.

Para ello se ha navegado por todo el campo de proyectos de plataformas como migranodearena.org o microdonaciones. hazloposible.org y cruzado la información obtenida con la facilitada directamente por estas plataformas, y de difícil acceso por nuestra parte. A continuación se muestran los principales resultados obtenidos de estas plataformas.

\subsection{Plataforma Microdonaciones. hazloposible.org}

Esta plataforma posibilita que personas individuales impulsen con pequeñas aportaciones económicas proyectos solidarios de distintas ONG. La plataforma propone proyectos, recibidos de ONG que estén constituidas legalmente en España y hayan sido declaradas de utilidad pública, y los donantes hacen sus aportaciones en aquellos que consideren de su interés. El $100 \%$ del importe recaudado se entrega a la $\mathrm{ONG}^{34}$. Es una iniciativa de la Fundación Hazloposible y cuenta en la actualidad con el apoyo económico de la Fundación Juan Entrecanales de Azcárate ${ }^{35}$ que cubre parte de los gastos operativos.

Se ha obtenido la información de 149 proyectos de los que 10 (60.9\%) han sido exitosos, que se han desarrollado desde Marzo de 2013 hasta junio de 2014. Este es el universo completo de proyectos realizados en esta plataforma en ese intervalo de tiempo ${ }^{36}$ siendo, todavía, reducido el número de elementos presentes en el mismo como para extraer conclusiones definitivas, muestra claramente la tendencia de crecimiento en número de proyectos y cantidad recaudada, si bien la escala media de los proyectos no ha sufrido una gran variación.

33 Verkami.com, Lanzanos.com microdonaciones.hazloposible.org y migranodearena.org han facilitado el acceso a sus datos.

${ }^{34}$ El único coste que se repercute al proyecto es el generado por la plataforma de cobros utilizada.

$35 \mathrm{http} / / /$ www.fundacionjea.org/

36 Resumen completo de los datos en http://bit.ly/cwd_soc_jul14 


\section{Tabla 1}

Evolución y caracterización de los proyectos realizados por microdonaciones.hazloposible.org

\begin{tabular}{|c|c|c|c|}
\hline & 2012 & 2013 & $2014^{*}$ \\
\hline N.o Proyectos & 31 & 85 & 23 \\
\hline$\%$ éxito & $61.2 \%$ & $80 \%$ & $69 \%$ \\
\hline Importe Total proyectos exitosos & $41.689 €$ & $116.434 €$ & $33.963 €$ \\
\hline Valor medio Proyectos finalizados con éxito & $2.194,16 €$ & $1.712,26 €$ & $2.122 €$ \\
\hline N. ${ }^{o}$ medio de mecenas & 31 & 28 & 29 \\
\hline Aportación media & $68,79 €$ & $60,68 €$ & $71,5 €$ \\
\hline $\begin{array}{l}\% \text { de fondos alcanzado sobre el objetivo en } \\
\text { proyectos finalizados exitosamente }\end{array}$ & $101,3 \%$ & $119,9 \%$ & $103,4 \%$ \\
\hline $\begin{array}{l}\% \text { de fondos alcanzado sobre el objetivo en } \\
\text { proyectos no exitosos }\end{array}$ & $11,76 \%$ & $12,68 \%$ & $5,33 \%$ \\
\hline
\end{tabular}

* primer semestre

Fuente: Elaboración propia.

La escala de los proyectos que se promueven a través de esta plataforma, está todavía lejos de representar una opción alternativa para la financiación de las ENL. A pesar de ello, con una aportación media cercana a la de las cifras del crowdfunding a nivel global, y pensando en alcanzar el número medio de mecenas existentes también a nivel global $(205)^{37}$ se observa un largo camino todavía por recorrer en esta plataforma.

Cabe resaltar que, a lo largo del tiempo de vida de esta plataforma, 20 ONG han presentado más de un proyecto.

\section{Tabla 2}

Número de entidades promotoras de más de un proyecto y número de proyectos acogidos por micronaciones.hazloposible.org

\begin{tabular}{cc}
\hline Número de ONG & Número de proyectos \\
\hline 15 & 2 \\
3 & 3 \\
1 & 6 \\
1 & 8 \\
\hline
\end{tabular}

Fuente: elaboración propia.

37 thecrowdfundingcentre.com 
Igualmente se ha registrado una participación reiterativa en los mecenas que han colaborado en la financiación de los proyectos presentados en esta plataforma.

\section{Tabla 3}

Número de mecenas recurrentes y proyectos en los que han participado.

\begin{tabular}{cc}
\hline Número de mecenas & Número de proyectos en los que participan \\
\hline 3.138 & 1 o más \\
388 & 2 o más \\
128 & 3 o más \\
65 & 4 o más \\
43 & 5 o más \\
\hline
\end{tabular}

Fuente: elaboración propia.

La información obtenida sobre la utilización de elementos que aporten calidad (videos, imágenes) y viralidad (comentarios, actualizaciones) a las campañas de crowdfunding muestran igualmente un crecimiento importante. Este dato debe tomarse con cierta cautela por haberse obtenido en un periodo de tiempo muy cercano al lanzamiento de la plataforma.

\section{Tabla 4}

Principales elementos cualitativos de microdonaciones.hazloposible.org

\begin{tabular}{lc}
\hline & $2013 / 2012$ \\
\hline Videos del promotor & $385 \%$ \\
Comentarios de los Usuarios & $177 \%$ \\
Actualizaciones en la página del proyecto & $600 \%$ \\
Galería de Imágenes & $405 \%$ \\
\hline
\end{tabular}

Fuente: Elaboración propia. 


\subsection{Plataforma migranodearena.org}

Se define como una plataforma de crowdfunding solidario cuyo objetivo es la recaudación de fondos a favor de las ONG, pretende acercar la solidaridad a los ciudadanos posibilitando a los mismos pequeñas aportaciones a favor de causas sociales. Esta plataforma ha sido creada por la Fundación Real Dreams ${ }^{38}$ y aporta el $100 \%$ del capital recaudado al proyecto ${ }^{39}$. En esta plataforma son las propias personas las que crean los proyectos a favor de ONG's concretas.

Se ha extraído la información de 1.695 proyectos de los cuales 216 $(12,75 \%)$ han concluido con éxito ${ }^{40}$. El primer proyecto data de septiembre de 2008 (importe medio de proyectos exitosos en este año $535 €$, n. $^{\circ}$ de mecenas medio 15, donación media $35 €$ ) y se han obtenido datos hasta el primer semestre de 2014 (importe medio de proyectos exitosos en este año $1.482 €, \mathrm{n} .{ }^{\circ}$ de mecenas medio 28 , donación media $52 €$ )

\section{Tabla 5}

Evolución y caracterización de los proyectos realizados por migranodearena.org

\begin{tabular}{lccccc}
\hline & 2009 & 2010 & 2011 & 2012 & 2013 \\
\hline $\begin{array}{l}\text { N.o Proyectos } \\
\text { \% éxito }\end{array}$ & 106 & 199 & 24 & 83 & 100 \\
$\begin{array}{l}\text { Importe Total proyectos } \\
\text { exitosos }\end{array}$ & $3.406,00 €$ & $19.303,00 €$ & $11.724,00 €$ & $111.371,00 € 238.215,00 €$ \\
$\begin{array}{l}\text { Valor medio Proyectos } \\
\text { finalizados con éxito }\end{array}$ & $121,00 €$ & $448,90 €$ & $781,00 €$ & $2.716,00 €$ & $3.842,00 €$ \\
N.o medio de mecenas & 4 & 9 & 13 & 15 & 59 \\
Aportación media & $31,00 €$ & $47,00 €$ & $60,75 €$ & $85,80 €$ & $64,00 €$ \\
\hline
\end{tabular}

Fuente: elaboración propia.

A pesar de la existencia, principalmente en los datos del año 2013, de valores extremos en el conjunto de datos, a los efectos perseguidos artículo estos no introducen una distorsión que cambien las conclusiones,

\footnotetext{
38 http://www.fundacionrealdreams.org/

39 El único coste que se repercute al proyecto es el generado por la plataforma de cobros utilizada.

40 La naturaleza de la plataforma que permite libremente la presentación de proyectos y el amplio plazo de realización de los mismos, condiciona este dato.
} 
sino más bien confirman la viabilidad de utilización del crowdfunding para proyectos de mayor entidad ${ }^{41}$ siempre que el impacto de los mismos lo justifique.

La propia plataforma ha facilitado información sobre el grado de interacción de los usuarios de la misma con los proyectos presentes en ella ${ }^{42}$. En todos ellos se observa una tendencia de crecimiento positiva, lo que está de acuerdo completamente con los resultados del estudio de thecrowdfundingcenter.com

Los siguientes gráficos se han obtenido del análisis de los proyectos finalizados con éxito en la plataforma migranodearena.org, en bloques trimestrales. Los datos han sido proporcionados por la propia plataforma ${ }^{43}$.

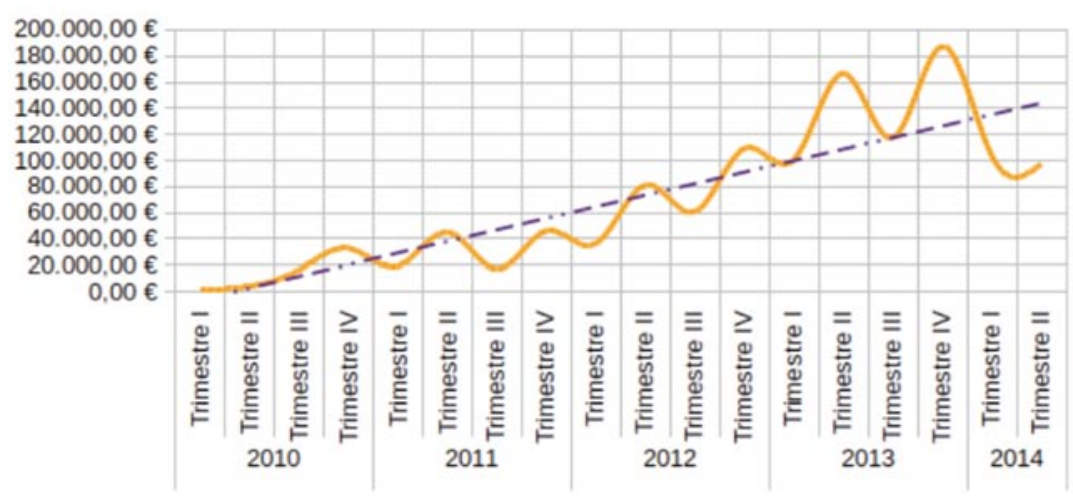

Fuente: Elaboración propia. Datos proporcionados por migranodearena.org

\section{Gráfico 1}

Cantidad recaudada trimestralmente

${ }^{41}$ Proyectos para la investigación del cáncer infantil de un volumen superior a los $50.000 €$

42 Resumen completo de los datos en http://bit.ly/cwd_soc_jul14

43 Resumen completo de los datos en http://bit.ly/cwd_soc_jul14 


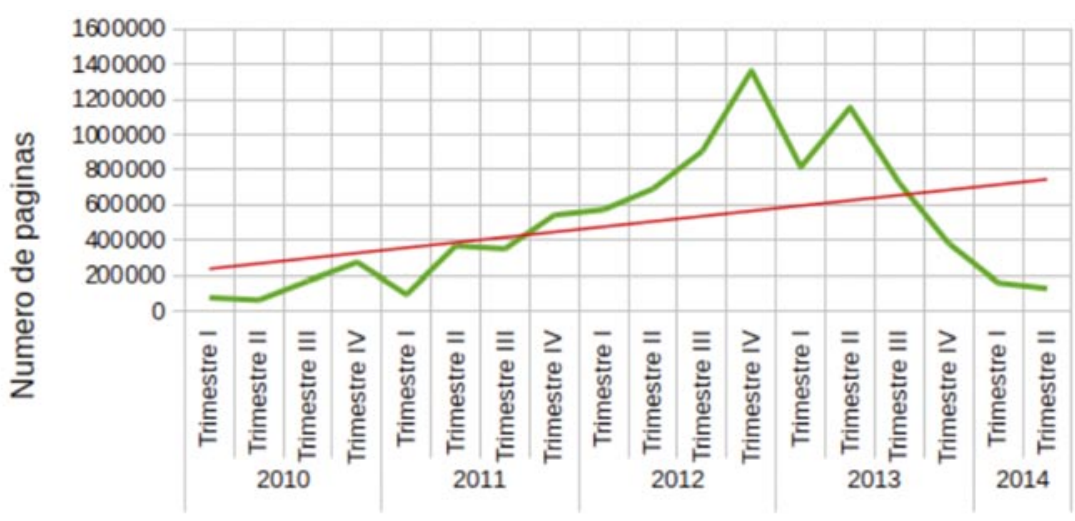

Fuente: Elaboración propia. Datos proporcionados por migranodearena.org

\section{Gráfico 2}

Número de páginas vistas

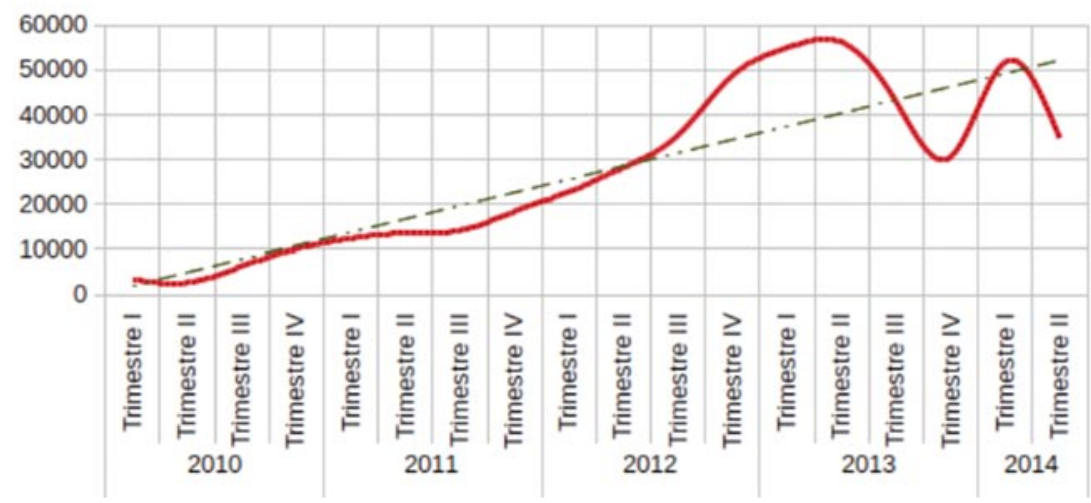

Fuente: Elaboración propia. Datos proporcionados por migranodearena.org

\section{Gráfico 3}

Número de visitantes únicos a la plataforma 


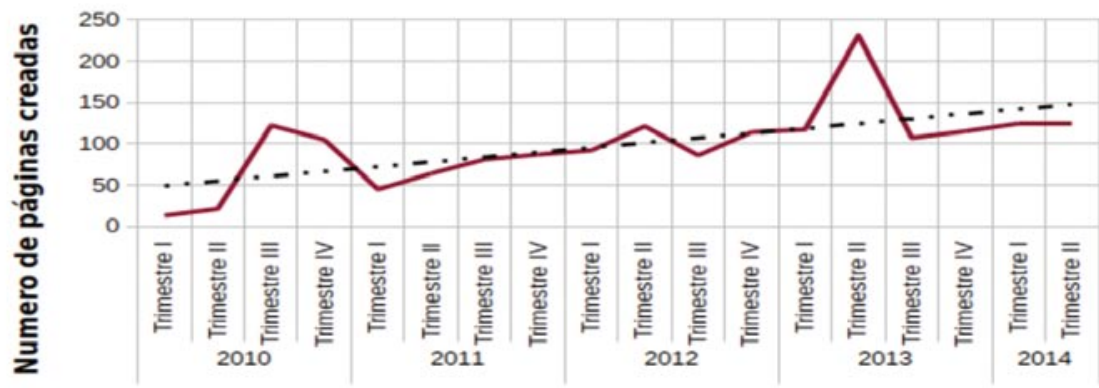

Fuente: Elaboración propia. Datos proporcionados por migranodearena.org

\section{Gráfico 4}

Número de páginas creadas

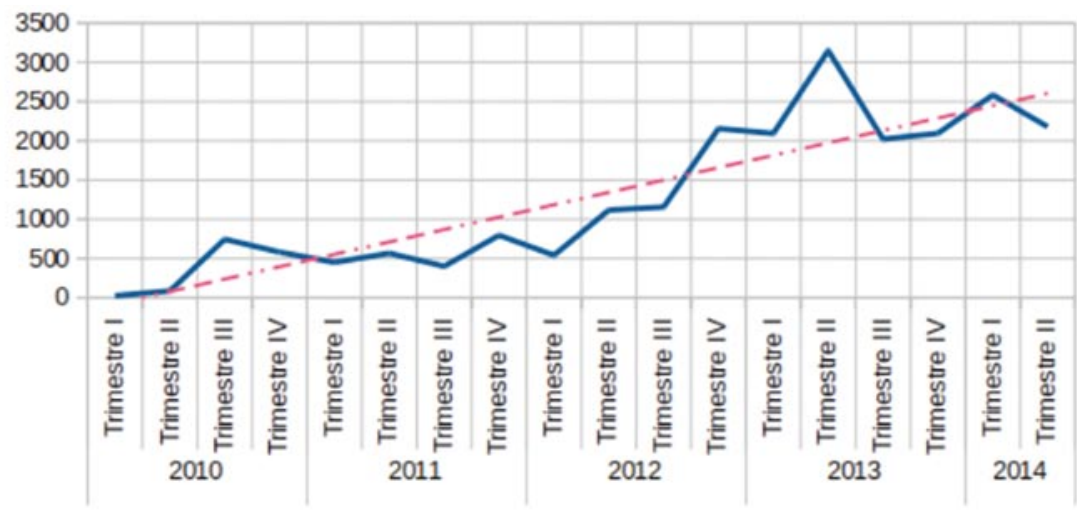

Fuente: Elaboración propia. Datos proporcionados por migranodearena.org

\section{Gráfico 5}

Número de donaciones 


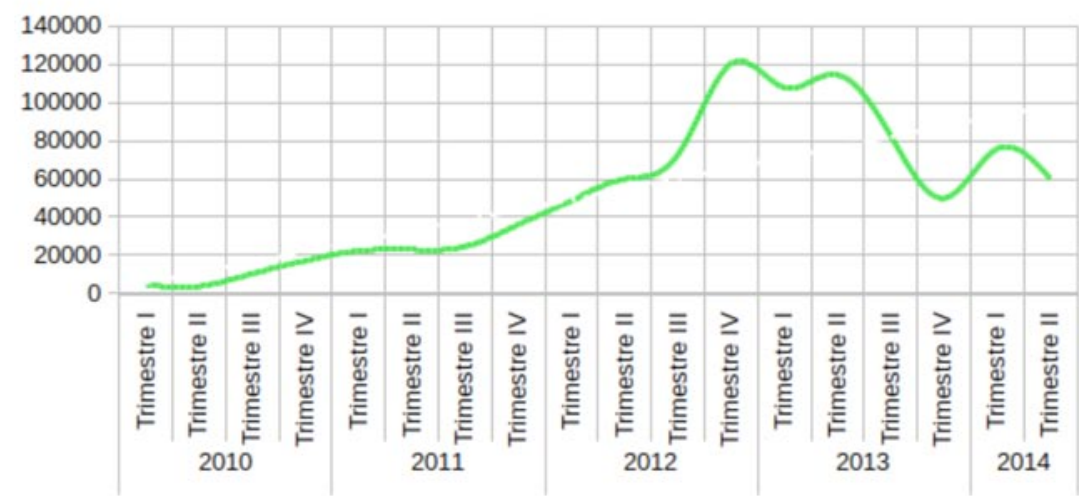

Fuente: Elaboración propia. Datos proporcionados por migranodearena.org

\section{Gráfico 6}

Número de visitas a la plataforma

\section{Conclusiones y retos}

El entorno social, político y económico en que tienen que desarrollar su actividad el SNL español en los inicios del siglo XXI, se encuentra profundamente marcado por los efectos de una prolongada crisis financiera. Sus primeras consecuencias, en el ámbito que nos ocupa en este artículo, han sido la implantación en diversos estados de políticas de recortes presupuestarios y austeridad en el gasto. Ambas realidades tienen una incidencia importante en el marco del estado del bienestar debido a las corrientes políticas que han gobernado la mayoría de los estados de la UE en los años de mayor azote de la crisis a la que nos referimos.

Con este escenario, el rol de las ENLs se torna trascendental ya que son ellas las que hacen frente a las necesidades sociales de gran parte de la población ante la retirada de las estructuras estatales. Es en este punto donde se produce un hecho paradójico con la desatención a este tipo de entidades en los presupuestos públicos, dejando al pie de los caballos a una, cada vez mayor, cantidad de personas cuya vida ha sido trastocada por los efectos de la crisis y que es ahora, en el tiempo que se encuentran en una situación personal crítica, cuando requieren de una atención adecuada para garantizar una cobertura adecuada de sus necesidades básicas.

Las tradicionales aportaciones de la empresa privada a este tipo de entidades se han visto igualmente reducidas por la propia coyuntura econó- 
mica. Muchas de éstas donantes habituales, han visto aparecer resultados negativos en sus cuentas de resultados y esto les ha impelido a abandonar sus aportaciones o colaboraciones con acciones sociales.

El nuevo escenario tecnológico y la transformación que este ha provocado en los procesos habituales de desarrollo vital del ciudadano medio, es la base sobre la cual se han desarrollado iniciativas diversas con el objetivo de posibilitar la financiación de proyectos concretos en diferentes ámbitos, entre ellos el social. Esto dota al SNL de nuevas herramientas de obtención de recursos financieros, de un modo distribuido, colaborativo, compartido y democrático, características inherentes a las nuevas tecnologías. Estos mismos principios se encuentran grabados en el ADN de la Economía Social , y muy particularmente las ENLs, aspecto que añade un punto más de interés para continuar en la investigación del desarrollo de estas nuevas estrategias de fundraising.

La nueva sociedad pluri-interconectada, pluri-informada y multi-nivelada merced a la web 2.0 , se revela globalizada y sensibilizada en pro de los ámbitos sociales, y genera nuevas herramientas de financiación para el Sector, como es el crowdfunding, o financiación en masa a través de internet. Esta nueva filantropía pone a disposición de las ENLs la construcción de un conjunto de relaciones de mayor profundidad con los donantes que llegan a comprometerse profundamente con las causas que apoyan. Las ENLs pueden ubicarse en este nuevo esquema como participantes en redes más complejas que solucionan necesidades, a través de una financiación más plural, menos dependiente y más sostenible.

El fenómeno del crowdfunding abre la puerta al abandono de la dependencia financiera, algo habitual en las ENLs, de un número reducido de donantes mediante la creación de colectividades afines, a las que va a acudir posteriormente par a la obtención de recursos financieros, y en ocasiones materiales y humanos, para el desarrollo de proyectos futuros.

La naturaleza propia de este fenómeno, facilita la diseminación de la misión y visión de las ENLs mediante la realización de campañas para la captación de fondos, facilitando igualmente la creación de comunidades virtuales dispuestas a dar soporte a modelos concretos de acciones en campos específicos. Una campaña de crowdfunding, más allá que para la mera obtención de recursos, tiene una incidencia importante en la difusión, no solamente del proyecto objeto de la campaña, sino también de la entidad que lo promueve. De este modo, se posibilita la construcción paulatina de una masa social con el tamaño suficientemente para darle soporte a largo plazo.

Los recursos que deben ser asignados, por parte de la ENL, para el desarrollo de esta estratégica de captación de fondos son mínimos debido, por una parte, al funcionamiento filantrópico de la mayoría de las platafor- 
mas de crowdfunding social ${ }^{44}$, y por otro a que las tareas de desarrollo de las campañas de crowdfunding van a poder ser asumidas por el mismo equipo humano que se ocupa de las herramientas sociales y la comunicación en las ENLs. Adicionalmente nos encontramos con un proceso, a priori, de diseño y validación del proyecto para, de este modo, garantizar en la medida de lo posible la ejecución del mismo en los términos en que es presentado en la plataforma correspondiente ${ }^{45}$.

El impulso que el fenómeno del crowdfunding, como alternativa a los métodos tradicionales de financiación, está provocando un crecimiento exponencial que conlleva la duplicación de su tamaño cada 60 días ${ }^{46}$.

En el ámbito social y solidario, personas particulares y pequeńos colectivos de la sociedad civil han visto en él la forma de obtener los recursos, no únicamente financieros, que hasta ahora constituían el principal obstáculo para el desarrollo de distintas iniciativas dirigidas a pequeños grupos de beneficiarios.

Plataformas exclusivas de crowdfunding social como son microdonaciones.hazloposible.org, migranodearena.org, teaming.net o worldcoo.com han desarrollados diferentes esquemas de financiación colectiva en torno a ENLs tanto para proyectos concretos como para un sustento continuo en base a donaciones periódicas. La innovación está presente en el crowdfunding social, y modelos como socialtocrowd.org ${ }^{47}$ que aportan un nuevo enfoque ${ }^{48}$, más allá de los valores financieros, abren espacios de viabilidad en el Sector. Otras plataformas como verkami.com, lanzanos.com, goteo.org, indiegogo.com, kickstarter.com no siendo exclusivas de proyectos sociales si ofrecen un tratamiento preferente a este tipo de proyectos con un cobro inferior de comisiones ${ }^{49}$.

La no existencia en los proyectos sociales de crowdfunding de grandes donantes, que puedan intentar influir en el camino a seguir por la entidad a la que están aportando sus fondos pese a no estar, probablemente, alineados con la idea original de la misma, constituye un paso hacia la in-

44 principalmente en modalidad de donación

45 La práctica totalidad de las plataformas de crowdfunding realizan un estudio de idoneidad de los proyectos previo a ser lanzados, pero una parte de las mismas permiten recabar la opinión de los potenciales financiadores de proyectos, coadyuvando, a través de una corriente de de feedback informativo, en su diseño y estructura. Este es el caso de las plataformas españolas Lanzanos o Verkami

46 http://startsomegood.com/

47 Actualmente en proceso de creación mediante una campańa de crowdfunding en goteo.org.

48 Plataforma para facilitar la solidaridad directa entre organizaciones y personas, sin la dependencia del dinero y generando un nuevo valor en la reutilización de las cosas.

49 Estas plataformas se financian principalmente mediante el cobro de un porcentaje de comisiones sobre los fondos recaudados en los proyectos que se gestionan a través de ellas. 
dependencia y la consistencia en valores y principios en torno a los cuales, con una buena gestión de las herramientas sociales, es posible construir una comunidad que soporte financieramente a las ENLs proporcionando al mismo tiempo un incremento de respaldo social a las mismas. De este modo el crowdfunding emerge como un elemento para afrontar estos dos principales retos de las ENLs.

Tanto desde el punto de vista cualitativo como cuantitativo, ambos analizados en el presente trabajo, se halla constatadas la importancia de factores como la confianza y la capacidad de construcción de comunidades alrededor de ella para incrementar la escala de los proyectos de ámbito social que se financien vía crowdfunding. Las herramientas sociales y una gestión adecuada de las mismas son una pieza clave de éxito.

Es evidente que las ENLs cuentan con nuevos instrumentos para la construcción de sus propias estrategias de financiación y ejecución de proyectos, y es importante tener en cuenta que, para su correcto desarrollo, el fenómeno del crowdfunding va a precisar de:

El desarrollo de una regulación acorde con la realidad actual de las ENLs y del marco tecnológico, social y económico en el que se desenvuelven cada día. Una ley del Mecenazgo adecuada que contemple exenciones fiscales adecuadas para impulsar la participación del donante, no únicamente por su implicación directa o indirecta con el proyecto a financiar, sino también por la obtención de ventajas fiscales por este hecho. En su defecto, la creación de una legislación específica para el sector que pueda suplir las deficiencias, desde la perspectiva del mismo, existentes en la actual ley del Mecenazgo.

Debe evitarse una postura aprovechada por parte de la administración en la cual, en base a la participación de la ciudadanía en la financiación de proyectos o planes sociales concretos, repliegue sus velas eludiendo sus responsabilidades en este campo. El crowdfunding no es, para nada, una herramienta para la transmisión de responsabilidades desde la administración a la ciudadanía, sino todo lo contrario. Con una regulación coherente con la realidad social de los tiempos actuales, puede llegar a entenderse como un primer paso hacia una participación directa de la ciudadanía en el diseño de las líneas estratégicas de acción, trazadas tradicionalmente por las administraciones públicas, en el diseño del Estado del Bienestar.

\section{Referencias Bibliográficas}

Adecco (2011): Annual Report 2011, Adecco Group Company. Agraval A., Catlini Ch., Goldfarb A. (2011): «The Geography of Crowdfunding» Universidad de Toronto http://ssrn.com/abstract=1692661 
Anteproyecto de Ley de modificación de la Ley 38/2003, de 17 de noviembre, General de Subvenciones.

BBVA (2013) Crowdfunding: A disruptive Technology for Commercial Banks en U.S. Economic Outlook, second Quarter 2013.

Belleflame,P. \& Lambert,T. \& Schwienbscher,A. (2010). "Crowdfunding: An Industrial Organization Perspective», Digital Business Models: Understanding Strategies, Paris, 25-26 junio, 2010.

Belleflamme, P. et al. (2014): «Crowdfunding: Tapping the Right Crowd», Journal of Business Venturing, 29, (5)585-609.

Belleflamme, P. et al. (2013): "Individual Crowdfunding Practices», Venture Capital,15, (4), 313-333.

Bradford,C.S. (2012): «Crowdfunding and The Federal Securities Law's», Columbia Business Law, 17, 48-62.

Boetree D.S., Seltzer T. (2009). «Dialogic strategies and outcomes: A review of environmental advocacy groups' Facebook profiles», Public Relations Review 35, 317-319.

Cejudi, A. \& Ramil, X. (2013) Crowdfunding. Financiación colectiva en clave de participación, Asociación Española de Fundraising.

Cumming, D.J. \& Leboeuf,G. \& Schwienbacher,A. (2014): «Crowdfunding Models: Keep-it-All vs All-or-Nothing», Paris Finance Association, diciembre 2014.

$\operatorname{COM}(2014) 172$ final «Unleasing the potential of Crowdfunding in the European Union», Comisión Europea.

Crowdsourcing. Org (2012): The Crowdfunding Industriy Report, 2012: Market Trends, Composition and Crowdfunding Platforms. http://crowdfundcapitaladvisors.com/resources.html

Davies R. (2014): Civic Crowfunding: Participatory Communities, Entrepreneurs and the Political Economy of Place, Massachussets Institute of Technology

Donath, J. (2008). «Signals in social supernets. Journal of Computer-Mediated Communication», International Communication Association, 13, 231-351.

Gajda, O. \& Mason, N. (2013): Crowdfunding for impact in Europe and the USA, Toniic y European Crowdfunding Network (ECN)

Gerber E. \& Hui J. \& Kuo P. (2011): "Crowdfunding: Why People Are Motivated to Post and Fund Projects on Crowdfunding Platforms», NorthWestern University Creative Action Lab, CSCW Workshop, 2012 .

http://www.juliehui.org/wp-content/uploads/2013/04/CSCW_Crowdfunding_Final.pdf

Gerber E. \& Hui J. (2013): «Crowdfunding;: Motivations and Deterrents for Participation", ACM Transactions on Computing_Human Interaction, 20, 6.

Gill, M. \& Flynn R. \& Reissing, E. (2005): "The governance self-assestement checklist:An instrument for assessing board effectiveness". Nonprofit Management and Leadership 15:271-294.

Greenberg J. \& Macaulay M. (2009): «NPO 2.0? Exploring the Web Presence of Environmental Nonprofit Organizations in Canada», Global Media Journal, Canadian Edition, 2, (1), 63-88. 
Greenerg M. , Hui J. y Gerber E. (2013): "Crowdfunding: A Resource Exchange Perspective" http://egerber.mech.northwestern.edu/wp-content/ uploads/2012/11/Crowdfunding_A-Resource-Exchange-Perspective_2013.pdf

Greenberg,M.D. \& Hariharan,K. \& Gerber,E. (2013): "Crowdfunding Support Tools: Predicting Success \& Failure», CHI 2013: Changing Perspectives, Paris, France.

Griffin,Z. (2012): "Crowdfunding: Feeling The American Masses» http://papers s.ssrn.com/sol3/papers.cfm?abstract_id=1928168

Hackler, D., Saxton, G.D. (2007) "The strategic use of information technology by non profit organizations: Increasing capacity and untapped potential», $\mathrm{Pu}$ blic Administration Review, 67, 474-87.

Hemer,J. (2011): "A Snapshot on Crowdfunding", Working Paper Leibniz Information Center for Economics, N. ${ }^{\circ}$ R2/2011. http://hdl.handle. net/10419/52302

Howe ( 2008) Crowdsourcing, why the power of the crowd is driving the future of business, Three Rivers Press, New York.

Huang, K. \& Davenport, L. (2011). "The Adoption of Social Network Sites among Public Relations Practitioners at Non-Profit Environmental Organizations", AEJMC Midwinter conference, Norman, U.S.A.

Kickstarter.com (2014) Creator handbook https://www.kickstarter.com/help/handbook

Hui, J.S. \& Gerber,E.M. \& Gergle, D. (2014): «Understanding and Leveraging Social Networks for Crowdfunding: Opportunities and Challenges», DIS 2014, Vancouver, 21-25 de junio.

Klaebe, K. (2012): How to Work The Crow: A Snapshot of Barriers and Motivations to Crowdfunding, Creative Industries Faculty, Queensland, University of Technology.

Lamber T. y Schwienbacher A. (2010): „An Empirical Analysis of Crowdfunding» http://www.crowdsourcing.org/document/an-empirical-analysis-of-crowdfunding-/2458

Lanzanos.com (2014): ";Por qué la gente financia proyectos vía crowdfunding?» http://www.slideshare.net/twomuchrs/conclusiones-por-qu-la-gente-financiaproyectos-va-crowdfunding

Lozano Benito, L. (2009) «Economía Social 2.0. Las redes socioeconómicas en el nuevo entorno de las TIC», XII Jornadas Nacionales de Investigadores de Economía Social: La Crisis como oportunidad para la economía social, CIRIECEspaña 2010.

Mills, C. \& Davies, W. (2012): Proyecto de una década Cooperativa, Grupo de Trabajo de Planificación de la Alianza Cooperativa Internacional, Centro de Empresas Mutualistas y Propiedad de los Empleados, Universidad de Oxford.

Mitra,D. (2012): "The Role of Crowdfunding in Entrepreneurial Finance», Delhi Business Review X, 13, 2, 67-72.

Morales, A.C. (2009): "Innovación abierta en el Tercer Sector. El modelo 2.0», Revista Española del Tercer Sector,13,17-38. 
Morales, A.C. \& Ariza- Montes J.A. \& Múñiz, J. (2012): «El emprendedor social y el E-empowerment de las redes sociales, 75 .

Mollick E. (2014):»The dynamics of crowdfunding: An exploratory study» Journal of Business Venturing, 29, 1 - 16

Mosseyev A. (2013):»Effect of Social Media on Crowdfunding Project Results» University of Nebraska - Lincoln

http://digitalcommons.unl.edu/cgi/viewcontent.cgi?article=1043\&context=busin essdiss

Murray, R. (2010) Co-operation in the Age of Google - A review for Co-operatives UK - draft for comments.

http://www.uk.coop/sites/default/files/uploads/attachments/co-operation_in_ the_age_of_google.pdf

Olson, D.E. ( 2000) «Agency theory in the non-profit sector:its role at independent colleges». Nonprofit and Voluntary Sector Quarterly, 29:280-296.

PETS (2012): Diagnóstico del Tercer Sector de Acción Social - II Plan Estratégico del Tercer Sector de Acción Social, Plataforma del Tercer Sector de Acción Social, Madrid.

Plataforma de ONGs de Acción Social (2013): II Plan estratégico del Tercer Sector de Acción Social 2013-2016, Plataforma de ONGs de Acción Social.

PwC (2014): La reacción del Tercer Sector al entorno de crisis, ESADE - Obra Social La Caixa.

PwC et al. (2014): "Estudio sobre el presente y futuro del Tercer Sector Social en un entorno de crisis, Fundación La Caixa, Instituto de Innovación Social de ESADE , Fundación de PwC.

Sajardo, A. et al (2007): Valoración económica del Voluntariado en la Comunidad Valenciana, Fundación de la Solidaridad y el Voluntariado de la Comunidad Valenciana, Tirant lo Blanch.

Sajardo, A. (2012): Análisis económico del Sector No Lucrativo. Aspectos económicos del voluntariado, $2^{\text {a }}$ edición, Tirant lo Blanch.

Sajardo, A. (2014a): «El nuevo Fundraising: la era de la sociedad mecenas'», conferencia pronunciada en las IV Jornadas de FEAFES-CV: Estado de Bienestar Social. Viabilidad, Valencia 13 de diciembre de 2013.

Sajardo, A. (2014b): Estudio sobre el crowfunding social en España, Fundación Manantial. Madrid.

Smith, D.H. (1993) «Public Benefit and member benefit nonprofit, voluntary groups». Nonprofit and Voluntary Sector Quarterly, Spring 1993, 22, (1), 53-68

Steinberg, S \& Demaria, R. (2012): The Crowdfundiong Bilble, J. Kimmich. startsomegood.com (2014) How it works http://startsomegood.com/HowItWorks

Tomczak,A. \& Berm,A. (2013): "A Conceptualized Invest Model of Crowdfunding», Venture Capital,15, 4, 335-359. - Ward, C. y Ramachandaran, V. (2010): "Crowdfunding the next hit: Microfunding Online Experience Goods». http://people.cs.umass.edu/ - wallach/workshops/nips2010css/papers/ward.pdf 


\section{Webgrafia}

Achieve (2013): The millennial impact reseach - Resumen ejecutivo. http://cdn.trustedpartner.com/docs/library/AchieveMCON2013/Research\%20 Report/Millennial\%20Impact\%20Research.pdf

AEFR (2011): II Estudio de "benchmarking" sobre la situación de la captación de fondos y la rendición de cuentas en las ENL en España, Asociación Española de Fundraising. http://aefundraising.org/

AEFR (2013): El Perfil del Donante en España - Resumen de Prensa Asociación Española de Fundraising. http://aefundraising.org/

Boshop, M. \& Green, M. (2009) Philanthrocapitalism: How giving can save the world www.philanthrocapitalism.netCMI (2013) - 2014 Non Profit Content Marketing Benchmarks, budgets and trends North-America Pub, Content Management Institute Blackbaud. http://contentmarketinginstitute.com/wp-content/uploads/2013/11/Nonprofit_Research_2014_CMI.pdf - Crowdsourcing. Org (2012): The Crowdfunding Industriy Report, 2012: Market Trends, Composition and Crowdfunding Platforms. http://www.crowdfunding.nl/ - NTEN \& COMMON KNOWLEDGE \& BLACKBAUD, (2012) 4th Annual Nonprofit SocialNetwork Benchmark Report, Common Knowledge http://www.nonprofitsocialnetworksurvey.com/ - Nah, S. \& Saxton, G. D. (2012). Modeling the adoption and use of social media by nonprofit organizations. New Media \& Society. http://arxiv.org/pdf/1208.3394.pdf - Tapscott, D. (2012) - Networks and philanthropy: Philanthropy in the age of networked intelligence

Alliance Magazine Diciembre 2012 http://www.alliancemagazine.org/en/content/ philanthropy-age-networked-intelligence

X-Net (2013): Expericienias de Crowdfunding en el Estado español y Cataluña http://whois--x.net/img/crowdfunding_cast.pdf

Zvilichovsky,D. \& Inbar,Y. \& Barzilay,O. (2004): «Playing Both of the Narket. Success and Reciprocity on Crowdfundig Platforms" http://dx.doi.org/10.2139/ ssrn.2304101

\section{Plataformas referenciadas}

http://goteo.org/

http://www.verkami.com/

http://www.lanzanos.com/

http://migranodearena.org/

http://microdonaciones.hazloposible.org/

www.worldcoo.com

http://startsomegood.com/

https://www.teaming.net/ 